The ribosomal RNA promoter of Acanthamoeba castellanii determined by transcription in a cellfree system

Preecha Kownin, Calvin T.lida, Sheryl Brown-Shimer and Marvin R.Paule

Department of Biochemistry, Colorado State University, Fort Collins, CO 80523, USA

Received 24 October 1984; Revised 2 February 1985; Accepted 6 August 1985

\begin{abstract}
The DNA sequences required for faithful initiation of ribosomal RNA transcription were determined. BAL-31 digestion was used to modify the rDNA template by introducing deletions from its $3^{-}$-and $5^{\circ}$ - ends. The resulting mutant DNAs were tested for template activity individually or in competition with wild type utilizing an in vitro transcription system from Acanthamoeba castellanii. The results identify the sequence extending from -31 to +8 to be absolutely required for transcription. In addition; when the region between -47 and -32 is left intact, transcription is augmented.
\end{abstract}

\title{
INTRODUCTION
}

In both prokaryotic and eukaryotic systems, the interaction of proteins with specific DNA sequences has been demonstrated to play an important role in the mechanism of RNA transcription and its regulation. Central among these, transcription initiation by RNA polymerases requires a specific sequence in the DNA termed the promoter. Determination of the DNA region making up the promoter is instrumental in determining mechanisms of RNA biosynthesis.

All prokaryotic promoters, as exemplified by E.coli, contain two conserved sequences, one around -10 (TATAATG) and the second at about -35 (TTGACA), that are recognized directly by the RNA polymerase (1). In contrast to prokaryotes, in eukaryotic systems there are three DNA-dependent RNA polymerases, each responsible for transcription of a different set of genes. The promoter sequences required for these three enzymes are different. The RNA polymerase II promoter consists of the sequence around the cap site plus two consensus sequences located upstream of the cap site: The TATA box or Goldberg-Hogness box resides around positions -20 to -30 , and the CAAT box in the -70 to -90 region. These two regions are required for both in vitro and in vivo transcription $(2,3,19)$. A further upstream sequence also stimulates in vivo transcription (4). In contrast, the RNA polymerase III promoter mainly resides within transcribed regions of the 
genes although flanking sequences have recently been implicated as well

(24). Specific transcription of tRNA genes requires two DNA sequence blocks between +8 and +19 and +52 and +61 , while 5S RNA synthesis is dependent on the region from +55 to +83 (5). The DNA sequences of the RNA polymerase II and III promoters are highly conserved among eukaryotic species.

The promoter for rRNA synthesis required by RNA polymerase I is found upstream of and slightly overlapping the transcription unit start site. The location of the promoter region varies in different organisms, residing between -43 and +20 in Drosophila (6), -142 and +6 in Xenopus $(7,8),-39$ and +11 in mouse $(9,10)$, and -158 and +18 in human (11). Though there is homology among closely related species $(12,20)$, in general, the RNA polymerase I promoter sequence is very poorly conserved. Further, in contrast to the polymerase II and III systems which can utilize heterologous components in vitro, the polymerase I system exhibits species specificity; the DNA template and transcription factors must be obtained from the same or closely related source (13). Therefore, a number of rRNA promoters are being examined in order to discern motifs with common functions rather than homologous primary sequences.

We are studying the control of rRNA synthesis in Acanthamoeba castellanil, a small free-living amoeba which undergoes cellular differentiation into a dormant cyst. Cessation of rRNA transcription is coordinated with differentiation, and the mechanism of polymerase I-mediated expression and its regulation is under study. Here, we report results of in vitro transcription studies showing that the rRNA promoter consists of a region extending from -31 to +6 that is required for faithful transcription. In addition, the efficiency of transcription is increased when the region from -32 to -47 is left intact, though this motif 18 not required for transcription in vitro.

\section{MATERIALS AND MBTHODS}

Construction of deletion mutants

The plasmids pSBX60 and pSBX601 were used for the construction of $5^{\circ}$ and $3^{-}$deletion mutants, respectively. (See RESULTS). Thirty micrograms of the plasmid was linearized by NruI restriction endonuclease (Bethesda Research Laboratory) under recommended conditions. After phenol extraction and ethanol precipitation, the DNA pellet was redissolved in 20 ul of deionized water. A $14 \mathrm{ul}$ aliquot of the NruI linearized plasmid was digested with 80 units of "slow" BAL-31 (International Biotechnologies, Inc.) 
under recommended conditions for 20 minutes. Twenty-five microliter aliquots were transferred at 1 min intervals into separate Eppendorf tubes containing 3 ul of $120 \mathrm{mM}$ EDTA, $\mathrm{pH} 8.0$ and $1 \%$ SDS and the BAL-31 was inactivated by heating at $65^{\circ} \mathrm{C}$ for 15 minutes. Af ter ethanol precipitation, the DNA pellet was redissolved in 15 ul of deionized water, and 5 ul of the resuspended DNA was treated with 2 units of T4 DNA ligase (BRL) in $20 \mathrm{ul}$ of $66 \mathrm{mM} \mathrm{Tris-HCl}, \mathrm{pH}_{0} 7.6,6.6 \mathrm{mM} \mathrm{MgCl}, 10 \mathrm{mM}$ dithiothreitol and $0.4 \mathrm{mM}$ ATP at $14^{\circ} \mathrm{C}$ for 16 hours. The resulting c1rcular DNA was used to transform E. coli strain $\mathrm{SK}_{1592}$ by $\mathrm{CaCl}_{2}$ treatment (14).

The extent of deletion in the resulting mutants was determined by restriction enzyme mapping and DNA sequence analysis (15).

\section{In vitro transcription assay}

The ability of the deletion mutants to direct faithful initiation of transcription was examined by the in vitro runoff transcription assay using heparin-Sepharose purified RNA polymerase I and partially purified transcription initiation factor from Acanthamoeba castellanil as described elsewhere (16).

\section{RESULTS AND DISCUSSION}

\section{DNA Templates}

A $2.3 \mathrm{Kbp}$ fragment of one of the $12 \mathrm{Kbp}$ rRNA repeat units was cloned between the EcoRI and HindIII sites of pBR322 to produce the clone pAr4 (21). The cloned fragment contains 600 bp of the nontranscribed spacer, the entire external transcribed spacer and the $5^{-}$-end of the 18S RNA coding sequence. A 915 bp thal fragment $(-420$ to +495 , where +1 is the first base of the primary transcript) contains the transcription start site (16, and unpublished) and all of the sequence necessary to direct faithful in vitro transcription of ribosomal RNA (rRNA) (16).

Clustered around the transcription start site are three XmaIII sites (at $-96,-54$ and +14 ). Two XmaIII-bounded fragments resulting from digestion of the pAr4/HhaI fragment were subcloned into the single XmaIII site in PBR322 to produce three plasmids: (Figure 1). (1) pSBX30 from the most upstream XmaIII fragment and containing rDNA sequence from -97 to -51 , (2) pSBX60 containing rDNA sequence from -55 to +19 (including the start site) oriented so that transcription of the rRNA gene occurs in a clockwise direction on the standard PBR322 map (22) and (3) pSBX601 containing the same insert as pSBX60 oriented in the inverted direction. 


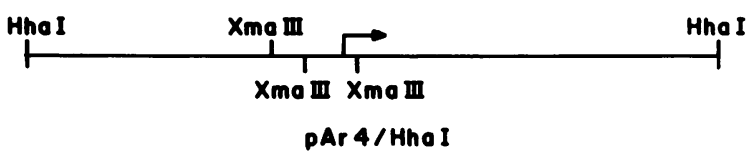

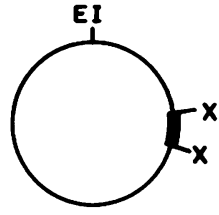

pSBX30

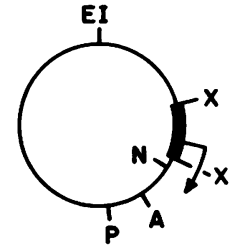

pSBX60

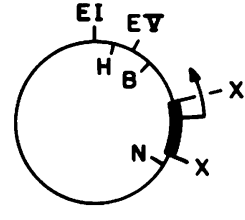

pSBX601

F1gure 1. Restriction endonuclease map of the pAr4/thal fragment, pSBX30, pSBX60 and pSBX60i. A = AvaI, B = BamHI, EI = EcoRI, EV = EcoRV, $H=H i n d I I I, N=$ NruI, $P=$ PvuII $X=$ XmaIII. In the lower half of the figure, the heavy line represents the XmaIII Inserted fragment, and the thin line pBR322.

\section{In vitro transcription assay}

We have developed an in vitro transcription system which accurately initiates synthesis at the start site on linear DNA templates (16). A runoff RNA is produced whose length is equal to the number of bases between the start site and the restriction endonuclease site used to truncate the template DNA (See Figure 2 for example runoffs from pSBX60 and pSBX601). The system requires the DNA template, highly purified RNA polymerase I from Acanthamoeba (23) and a partially purified auxiliary protein preparation containing proteins needed to direct the polymerase to the start site of the transcription unit.

\section{Lack of Requirement for far upstrean sequences}

Plasmid PSBX30 is inactive as a template (data not shown); the -55 to +19 clones PSBX60 and pSBX601 are capable of directing the synthesis of runof $f$ transcripts in the in vitro transcription system suggesting that only this limited sequence is necessary for faithful initiation (Figure 2) (16). However, it is not clear from these results alone that the limited sequence is as efficient as templates which include sequences from further upstream. Therefore, we have utilized a competition assay to test the efficiency of these minimal templates against a template which contains wild type sequence extending to -420 , the pAr4/HhaI fragment. In the assay, equimolar amounts of a BamHI/AvaI fragment of pSBX60i (which yields a 577 base runof $f$, Figure 


\section{Runoffs}

\section{$\begin{array}{llllllll}M & 1 & 2 & 3 & 4 & 5 & 6 & 7\end{array}$}

A. $\mathrm{pSB} \times 60$
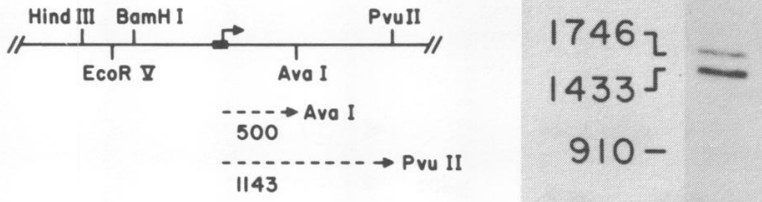

14335

$910-$

\section{$659-$}

B. $p S B \times 60 i$
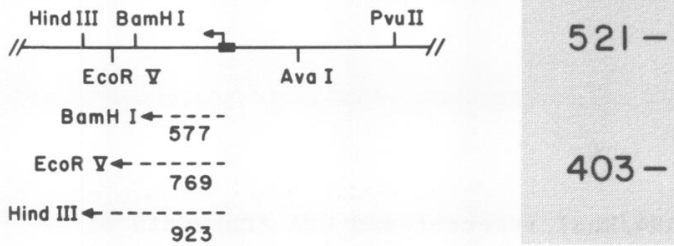

$403-$

Figure 2. A. Restriction endonuclease map and sizes of the runof $f$ transcripts from pSBX60. B. Restriction endonuclease map and sizes of the runof $f$ transcripts from $p S B \times 601$. C. Runoff transcription assay from linearized PSBX60 and PSBX601. lane 1. PBR322/AvaI, 2. pSBX60/AvaI, 3. pSBX60/PvuI I, 4. pBR322/BamHI, 5. pSBX601/BamHI, 6. pSBX601/EcoRV, 7. PSBX601/HindIII.

2) is mixed with the pAr4/Hhal fragment (which yields a 495 base runoff). The transcription assay is initiated by the addition of RNA polymerase I, transcription initiation factors and nucleoside triphosphates. Comparison of the intensities of the 577 base and 495 base transcripts in lane 3 of Figure 3 shows that the additional 365 base pairs of wild type sequence in the pAr4/Hhal fragment have no significant effect on the efficiency of the template. Similar results were obtained with a fragment from pSBX60 (Figure 3, lanes 4 and 5). In both experiments, the longer transcripts ( 577 and 1143) have a higher intensity in the autoradiogram than the shorter (495) because on a per molecule basis they contain more $\left[{ }^{32} \mathrm{P}\right]-\mathrm{CMP}$. Thus, it is clear that for in vitro transcription, only upstream sequences closer than -55 are needed for optimal transcription initiation. 


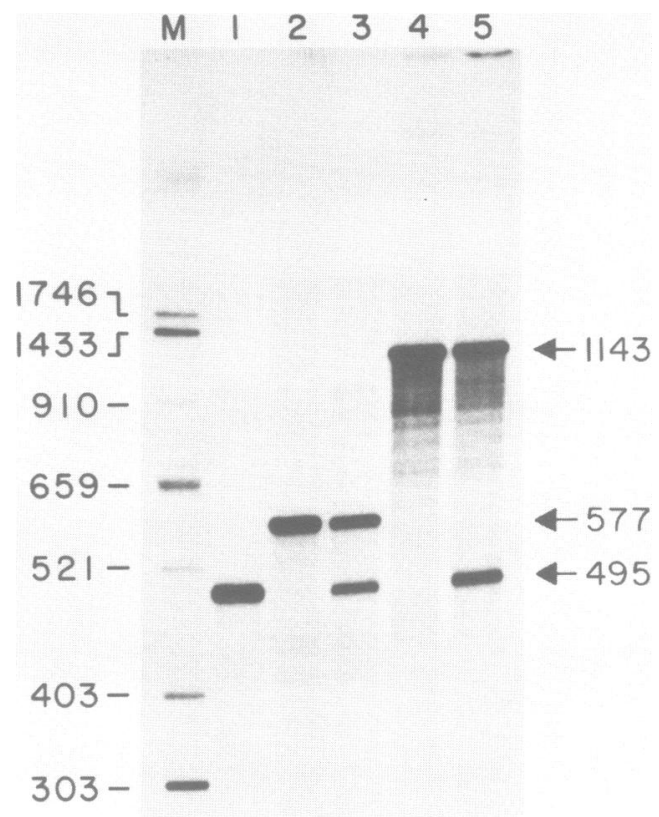

Figure 3. Competition between pAR4/HhaI fragment and DNA fragments of pSBX60 and pSBX601. Equimolar amounts of DNA templates were utilized in the in vitro transcription assay. lane 1. pAr4/HhaI ( 915 bp total length, runof $f=495$ nucleotides), 2. pSBX60i/BamHI-AvaI (1057 bp total length, runoff $=577$ nucleotides), 3. pAr4/HhaI and pSBX601/BamHI-AvaI, 4. pSBX60/BamHI-PvuII ( 1760 bp total length, runof $f=1143$ nucleotides), 5. pAr4/HhaI and pSBX60/BamHI-PvuII.

\section{Effect of $5^{-}$-deletions}

BAL 31 has been used to produce a series of deletions upstream of the start site (Figure 4A and Table I). PSBX601 was linearized with NruI, digested with BAL-31, a $5^{-}$and $3^{-}$exonuclease, and the resultant series of nested deletion mutants ligated, used to produce E. coli SK1592 transformants, and individual deletion mutants cloned and sequenced. The deletion containing plasmids were linearized with BamHI and tested as templates in the in vitro transcription system. The results are shown in figure 4A. All templates containing sequences downstream of -31 are active in transcription. (Deletions are named as the last deleted base. Thus 5-d1-32 has the same sequence as the wild type from -31 downstream.) However, when the upstream sequence has been deleted to -26 , the template no longer supports faithful infiation. This identifies the upstream border of the core promoter as between -31 and -26 . Since -31 is the maximum possible 
A. 5'-Deletion Mutants

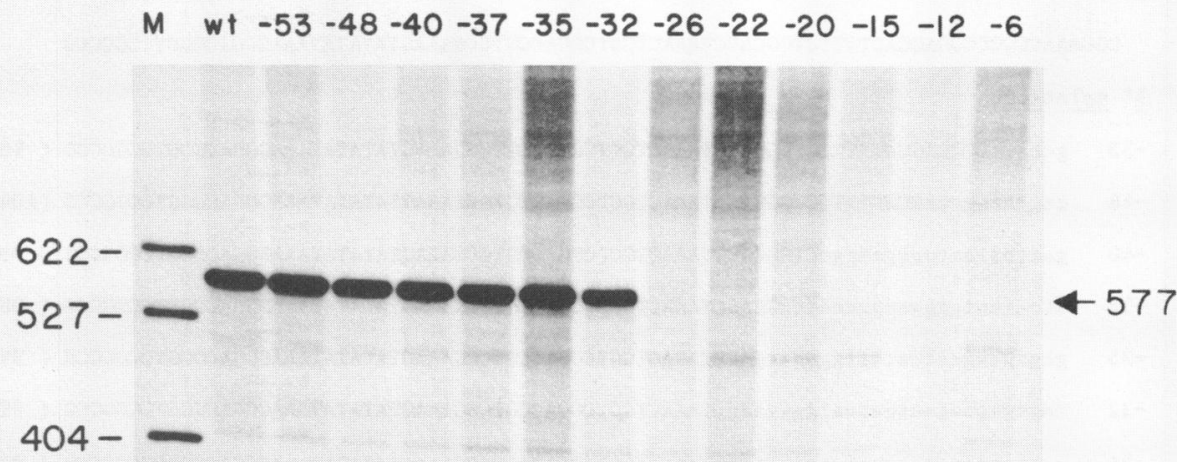

B. 3'-Deletion Mutants

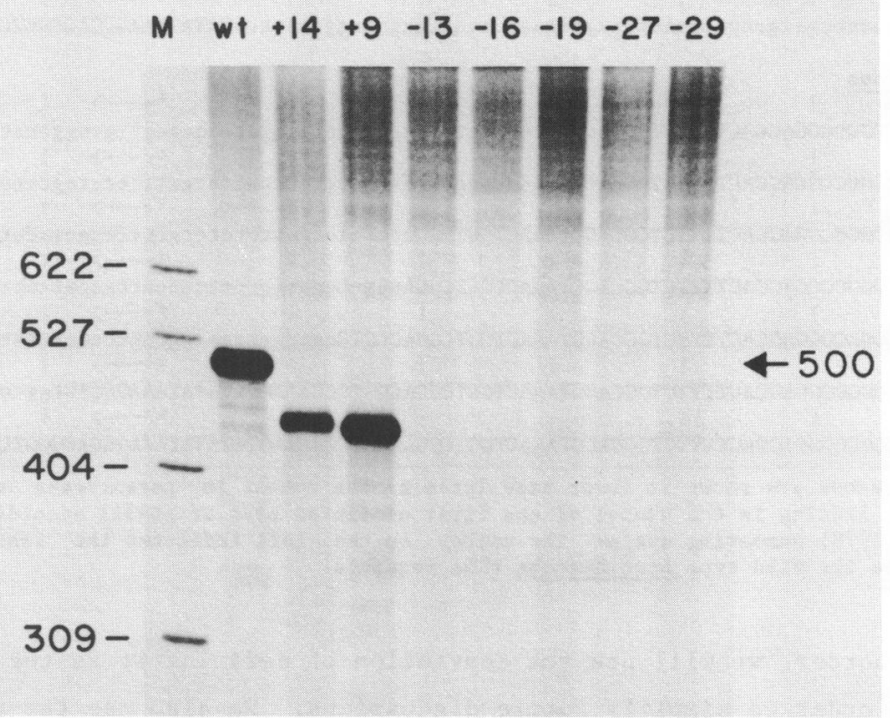

Figure 4. Transcription of wild type (wt), $5^{\circ}$ and $3^{-}$deletion mutants of Acanthamoeba ribosomal DNA. Each assay contained $0.1 \mathrm{ug} / 25 \mathrm{ul}$ of DNA template. A. $5^{-}$-deletion mutants. pSBX601 and mutants were truncated with BamHI. B. 3-deletion mutants. pSBX60 and mutants were truncated with Ava I. The numbers above the lanes correspond to the extent of deletion (Table I). 
TAUE I

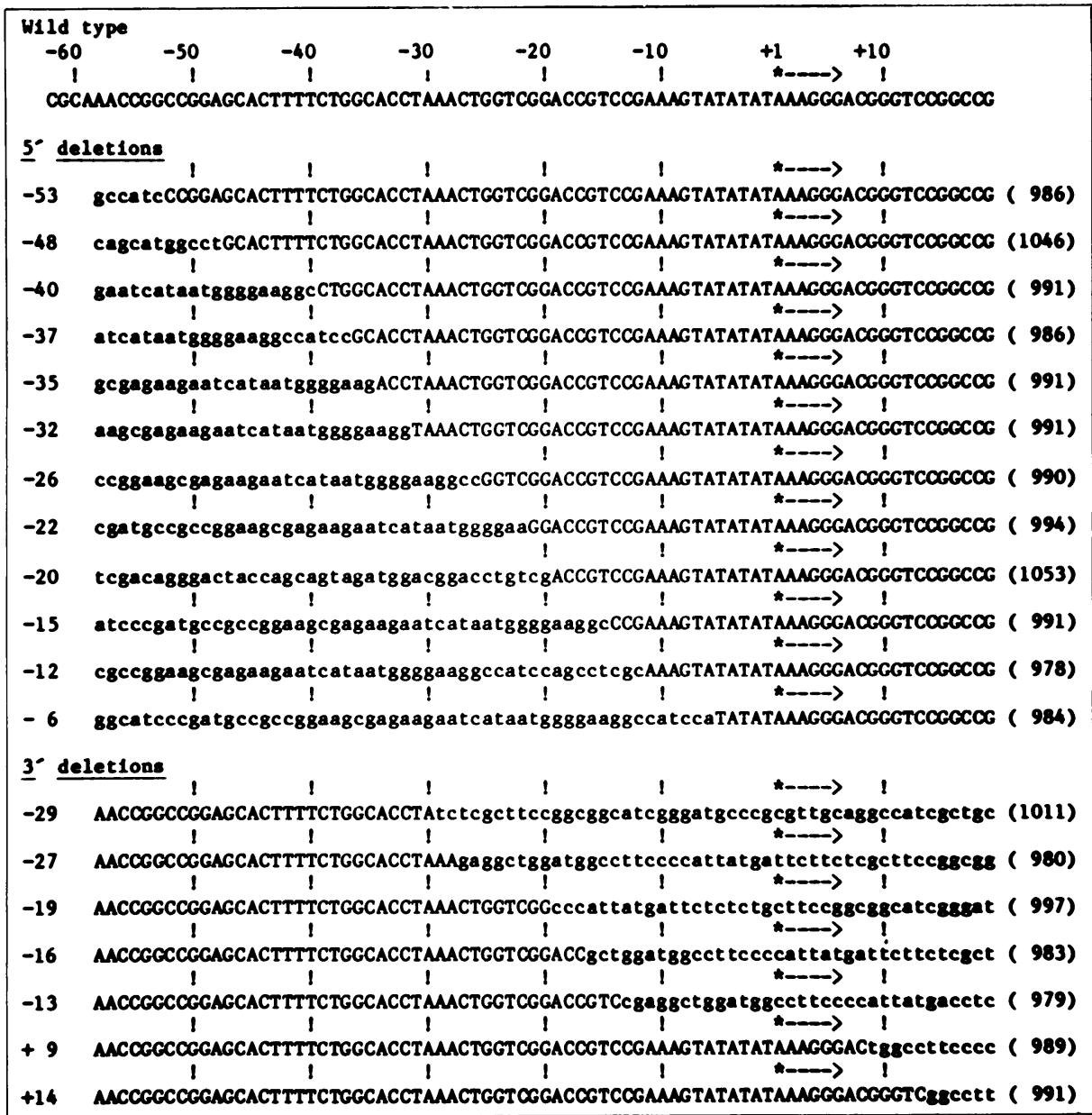

Vector sequences are shown in lower cese letters. The number in parentheses on the right end of each lleting 1s the number of the flret undeleted base of pBR322 according to the sutcllffe (1978) numberine syeten. The number on the left Indicates the last base palr deleted from the wild type Acentherenbe rRNA sequence.

upstream border, we will use the convention of defining it as the upstream border in order to simplify future discussions. We also use the term "core promoter" to designate those minimal sequences necessary to direct faithful initiation in our in vitro transcription system (see below).

Effect of $3^{-}$deletion

The plasmid pSBX60 was linearized with NruI and used (as described above) in the production of a series of $3^{-}$-deletion mutants. The resulting plasmids were linearized with AvaI and then tested for the ability to serve 
as templates to support faithful transcription in vitro. Deletions from the $3^{-}$side up to position +9 did not affect the template activity (Figure 4B). Deletion to position -13 completely eliminated the template activity, despite the presence of the sequence between -13 and -55 . These results define the downstream border of the promoter as +8 . There is a large imprecision in this downstream border because of a lack of deletions between +9 and -13 . We have screened over 300 BAL-31 deletion mutants from three separate digestions and have not found any additional constructs with end-points between +9 and -13 . Apparently BAL-31 rapidly traverses this very AT-rich region (74\%) producing mainly end-points on either side. Point mutations are being used to better locate the downstream border of the promoter.

Since deletions produced by the technique used here juxtapose different vector sequences adjacent to the deletion endpoints, there is the potential for vector sequences to effect transcription efficiency in the mutant constructs. Again, point mutation will eliminate this problem. However, we note that the endpoints of many of the deletions are very similar or identical (Table I) and thus the constructs have almost the same flanking vector sequences. Two different preparations of the $3^{-} d 1+14$ mutant have exhibited template activities lower than either the wild type or the +9 $3^{-}$-deletion. This may result from an inhibitory effect of flanking vector sequences in the $3^{-} d 1+14$ mutant or to a stimulatory effect of vector sequences on the $3^{-} d 1+9$ mutant.

\section{Competition between deletion mutants and wild type}

Mixing assays were performed by incubation of equimolar amounts of two different DNA templates in the in vitro transcription assay. When two wild type DNAs were added simultaneously, equal levels of specific transcription resulted, (Figure $5 \mathrm{~A}$, lane $\mathrm{c}$ ) indicative of the ability of the templates to compete for the necessary transcriptional components. Deletions from the $5^{-}$ side of the template to -53 , (lane d) and -48 (lane e) had little adverse ef fect on specific transcription when compared to the wild type. However, as the $5^{-}$upstream sequence was deleted to $-40,-37,-35$ and -32 , (1anes $\mathrm{f}-1$, respectively) the ability of these templates to fully compete with the wild type DNA was diminished. Transcription of the wild type DNA (923 nucleotides) increases noticeably when competed with the latter deleted templates. These differences are seen fairly consistently in repetitive assays. The deletions further downstream to $-26,-22,-20,-15,-12$ and -6 showed no transcriptional activity and the activity of the wild type 


\section{Nucleic Acids Research}

A. Competition: 5'-Deletion Mutants

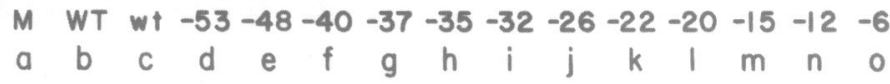

$622--$

$527--$

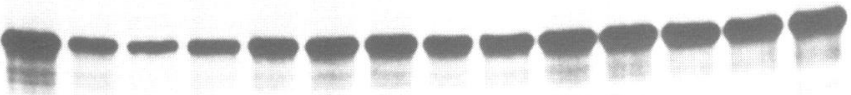

$404--$

B. Competition: $3^{\prime}$-Deletion Mutants

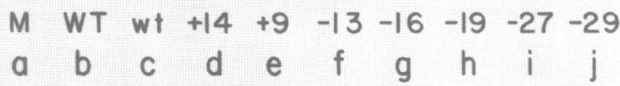

$622-$

$527-$

$\longrightarrow \rightarrow-577$

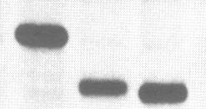

$\leftarrow 500$

$404-$

$309-$

Pigure 5. Competition between deleted and nondeleted templates.

Equimolar amounts of the deleted and nondeleted templates were utilized in the In vitro transcription assay. Each assay contained 0.1 ug/25ul of each DNA template. A. Competition of $5^{-}$-deletion mutants. lane b: pSBX60i/HindIII (wt). lanes c-o contained 0.1 ug of pSBX60i/HindIII (runoff $=923$ nucleotides) and the competitor DNAs truncated with BamHI 
(runoff $=577$ nucleotides). wt $=$ pSBX601/BamHI as competitor. The numbers above lanes $d-0$ correspond to the extent of $5^{\circ}$ deletion in the competitor DNA. B. Competition of $3^{-}$-deletion mutants. lane b. pSBX601/BamHI. lane $c-j$ contained 0.1 ug of pSBX601/BamHI (runoff $=577$ nucleotides) and the competitor DNAs truncated with AvaI (runof $f$ variable in size). wt $=\mathrm{pSBX60/AvaI}$ as competitor. The numbers above the lanes $d-j$ correspond to the extent of $3^{\circ}$-deletion in the competitor DNA.

template increased slightly (lanes $j-0$ ). These experiments define an upstream sequence motif, -32 to -47 , which is not required for transcription, but which stimulates transcription from the core promoter in cis. Its ef fect is only observed when the deleted template is competing with a "wild type" template.

Templates deleted from the $3^{-}$side to +9 show similar activities to the undeleted wild type pSBX60i/BamHI (Figure 5B, lanes c-e). However, further deletion abolishes all specific transcription; eg. deletions to $-13,-16$ and -19 in which the start region has been removed, produce no runof $f$ transcripts (Figure 5B, lanes $f-h$ ). Similarly, deletion to -27 and -29 are not transcribed and transcription of the wild type DNA is slightly increased over that in the other competitions (lanes $i$ and $j$ ).

In summary, the sequence requirement for faithful transcription initiation in vitro extends from -47 to +8 and can be subdivided into two domains: One of these, located between -31 and +8 is absolutely required for transcription. The second region, between -32 and -47 , enhances the efficiency of transcription but is dispensable for specific inftiation.

Using competition-prebinding assays (18), we have shown that the second, upstream sequence (motif B) in Acanthamoeba functions in stabilizing a preinitiation complex between one or several auxiliary proteins and part of the core promoter sequence. If motif $B$ is deleted, only a transient complex forms between the transcription initiation factor(s) and the template. This finding explains why, in the competition experiments reported here, templates deleted in motif $B$ are less effective templates than wild type. Wild type DNAs containing both motifs sequester transcription initiation factor(s) in stable complexes thus decreasing the concentration of avallable factor(s) for other templates deleted in motif B. It should be noted that the sequence ACTTT within motif B is similar to the conserved mammalian sequence, ATCTTT, which is in the -43 to -33 region of mouse, rat and man (12). The deletion of this conserved sequence lowers the efficiency of template activity in mouse as well as in Acanthamoeba $(9,10)$, suggesting a similar functional role for this region in mammals. 


\section{ACKNOWLEDGEAENTS}

Supported by grant GM26059 from the U.S. Public Health Service.

\section{REFERENCES}

1. Rosenberg, M and Court, D. (1979) Ann. Rev. Genetics 13,319-353.

2. Breathnach, R. and Chambon, P. (1981) Ann. Rev. Biochem. 50,349-383.

3. McKnight, S. L. and Kingsbury, R. (1982) Science 217,316-324.

4. Enhancers and Eukaryotic Gene Expression (Glutzman, Y. and Shenk, T., eds.) Cold Spring Harbor Press, Cold Spring Harbor, New York (1983).

5. Ciliberto, G., Castagnoli, L. and Cortese, R. (1983) Curr. Topics Dev. Biol. 18,59-87.

6. Kohorn, B. D. and Rae, P. M M. (1983) Nature 304,179-181.

7. Moss, T. (1982) Cel1 30,835-842.

8. Busby, S. and Reeder, R. (1983) Cell 34,989-996.

9. Yamamoto, 0., Takakusa, N., Mishima, Y., Komirami, R. and Muramatsu, M. (1984) Proc. Nat1. Acad. Sc1. USA 81,299-303.

10. Grunmt, I. (1982) Proc. Natl. Acad. Sci. USA 79,6908-6911.

11. Learned, R. M., Smale, S. T., Haltiner, M. M. and Tjian, R. (1983) Proc. Nat1. Acad. Sc1. USA 80,3558-3562.

12. Financsek, I., Mizumoto, K., Mishima, Y. and Muramatsu, M. (1982) Proc. Natl. Acad. Sc1. USA 79,3092-3096.

13. Grummt, I., Roth, E. and Paule, M. R. (1982) Nature 296,173-176.

14. Dagert, M. and Ehrlich, S. D. (1979) Gene 6,23-28.

15. Maxam, A. M. and Gilbert, W. (1980) Methods in Enzymology 65,499-559.

16. Paule, M. R., Iida, C. T., Perna, P. J., Harris, G. H., Brown Shimer, S. L. and Kownin, P. (1984) Biochemistry 23, 4167-4172.

17. Skinner, J. A., Ohrlein, A., and Grummt, I. (1984) Proc. Natl. Acad. Sci. USA $81,2137-2141$.

18. Iida, C., Kownin, P., Paule, M.R. (1985) Proc. Natl. Acad. Sci. USA (in press).

19. Hen, R., Sassone-Corsi, P., Corden, J., Goub, M.P and Chambon, P. (1982) Proc. Nat1. Acad. Sci. USA 79,7132-7136.

20. McStay, B. and Bird, A. (1983) Nucleic Acids Res. 11,8167-8181

21. D`Alessio, J.M., Harris, G.H., Perna, P.I. and Paule, M.R. (1981) Biochemistry 20,3822-3827.

22. Sutcliffe, J.H. (1978) Cold Spring Harbor Symp. Quant. Biol. 43,77-90.

23. Spindler, S.R., Duester, G.L., D’Alessio, J.M. and Paule, M.R. (1978) J. Bio1. Chem. 253,,669-4674.

24. Wilson, E. T., Larson, D., Young, L. S. and Sprague, K. U., J. Mol. Biol. (in press). 